\title{
Rising Above the Quantum Noise
}

\author{
The control of molecular-level quantum effects in artificial photosynthetic \\ membranes is a powerful tuning knob for optimizing long-range energy \\ transport, according to a theoretical study.
}

\author{
By Elisabetta Collini
}

$\prod$ he idea of engineering artificial molecular devices that mimic natural photosynthesis dates back to 1912, when it was first proposed by Italian photochemist Giacomo Ciamician [1]. However, the concept didn't move forward then because it was unclear how natural photosynthesis worked at the molecular level. More than a century later, experimental and theoretical advances are allowing researchers to fill this knowledge gap, and the improved understanding of the natural process is leading to the design of artificial molecular complexes that can efficiently capture the energy from sunlight

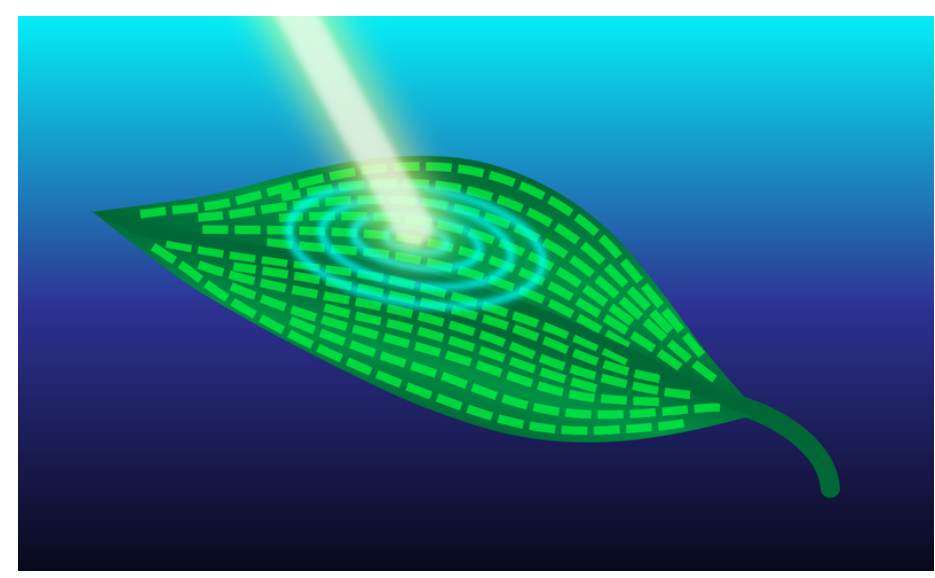

Figure 1: Artistic rendition of an artificial, bioinspired photosynthetic membrane. Mattioni and colleagues demonstrate that coherent quantum dynamics, even if localized within the unit cells that make up the membrane, can dramatically affect the transport of energy over spatial scales much larger than the unit cells.

Credit: APS/Alan Stonebraker
$[2,3]$. In this context, Andrea Mattioni of Ulm University, Germany, and colleagues have identified design principles for engineering light-harvesting membranes whose performance can be optimized by controlling quantum dynamics at the molecular level [4]. Such design principles are based on the counterintuitive result that, even at room temperature, quantum effects can be harnessed to optimize energy transport (Fig. 1). Specifically, the researchers' calculations suggest that the temporal and spatial scales of energy transfer in these membranes can be tuned by controlling how excitons-collective electron-hole states that dominate energy transport in these systems-become delocalized over the unit cells that make up the membranes.

The involvement of quantum mechanisms in energy transport in natural light harvesting is one of the most elusive and exciting aspects emerging from the study of photosynthesis. These ideas recently became testable thanks to the emergence of femtosecond multidimensional spectroscopy-a technique that can probe how quantum coherences propagate across different parts of a molecular system. These experiments have sparked a lively and yet-unresolved debate about the impact of quantum effects on energy transfer and light harvesting [5]. An outstanding question is whether quantum coherence can be engineered into artificial systems designed to control energy migration over macroscopic distances at room temperature.

To explore this possibility, Mattioni and his colleagues investigated a material whose modular architecture is inspired by that of certain biological light-harvesting complexes. Such complexes are made of multiple unit cells, each comprising 


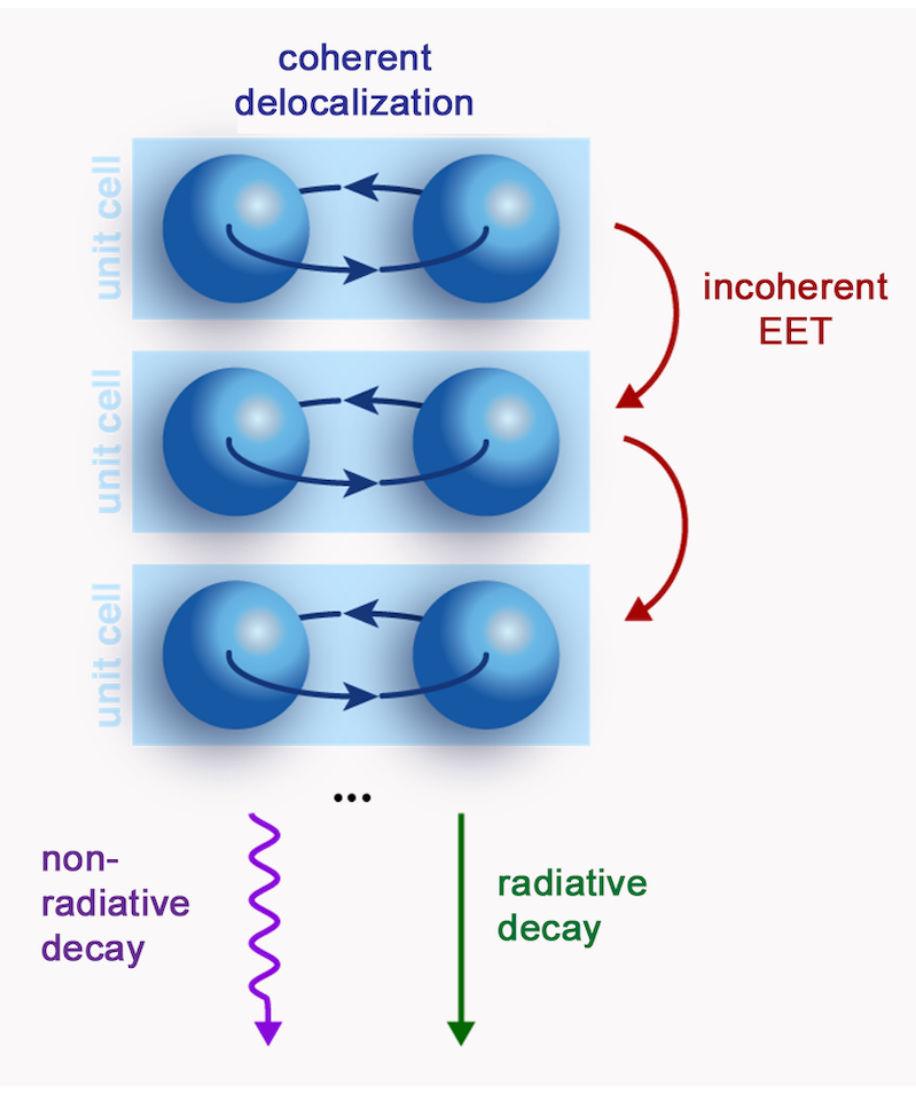

Figure 2: Sketch of the primary relaxation processes in a system described as an ensemble of unit cells, each formed by multiple pigments (blue spheres). The quantum coupling (dark blue arrow) among the pigments causes the excitons to be coherently delocalized over the cell, while energy transfer among different cells is governed by incoherent electron energy transfer (EET). Mattioni and colleagues demonstrate that quantum dynamics occurring within the pigments can be optimized to reduce the main dissipation pathways - both radiative (green) and nonradiative (purple)-that compete with and reduce the overall energy transfer efficiency.

Credit: E. Collini/University of Padua

several electronically coupled pigments. This coupling promotes the formation of excitons that can be delocalized over the entire cell [6]. Through self-aggregation, these unit cells form larger membrane architectures. In this modular structure, transport within unit cells is governed by the coherent quantum dynamics of delocalized electronic excitation. But over longer-range distances, the close contact with other unit cells allows energy transfer via classical, or incoherent, energy-transfer mechanisms (Fig. 2).

By using an exactly solvable model, the researchers demonstrate that the quantum properties developing within each unit cell have a substantial impact on how the energy of electrons-initially excited by absorbed photons-propagates through the entire system. The calculations indicate that the coherent exciton delocalization over several pigments within each unit cell gives rise to a peculiar energy-level structure. Such a structure results from contributions of both high-energy states that are "dark"-they can't be coupled to photons-and low-energy, "bright" excitons. Both dark and bright states are involved in energy transport, and their contribution strongly depends on the excitonic coupling between pigments.

The team analyzed the relationship between the excitonic states and the energy transport among different units. They found that, on one hand, energy transfer between unit cells can be much faster if it proceeds through the dark states than if it does so through the bright states, especially when the packing density of unit cells is large. This speedup stems from the delocalization properties of such states. On the other hand, relaxation within the unit cell-whereby energy is transferred from dark to bright excitons-increases the time window and distance over which energy can be transferred. This gain is due to the fact that bright excitons are less likely to lose energy by undergoing nonradiative decay (relaxation not associated with the emission of light). The researchers show that the balance of these two effects can be tuned to optimize both the speed and the spatial range of classical energy transport over many unit cells.

The picture emerging from these results is that the coherent delocalization of electronic excitations over just a few pigments within each unit cell is an effective "tuning knob" for controlling the large-scale properties of multichromophore molecular materials. Such a picture could explain the long-range excitation diffusion previously observed in nanoengineered, quasi-1D assemblies of closely packed light-harvesting complexes known as LH2 [7]-a behavior that has so far defied explanation. Mattioni and colleagues show that these devices can be so efficient because they operate in a parameter regime that sustains both the enhanced dark-state transfer mechanism and the shielding action of bright excitons against nonradiative decay. 
These results clear some doubts about the possibility of harnessing quantum coherence effects to boost the efficiency of energy transport in artificial photosynthetic membranes at room temperature. Until now, the main reason for skepticism was related to the limited temporal and spatial extent of quantum coherences, which are easily washed out by the coupling to external degrees of freedom (often referred to as "noise"). Most researchers would assume that this noise would kill any prospects of harnessing quantum coherence in engineered devices. But the work of Mattioni and co-workers indicate that this noise limitation can be overcome, as energy transfer in these modular systems is related to, and can be controlled through, excitonic delocalization, even if this delocalization is restricted to a few pigments within a unit cell.

Interestingly, the researchers' calculations indicate that the same noise that limits the persistence of coherence in space and time can be turned into a beneficial resource-an idea that some of the researchers had previously suggested [8]. In the new work, Mattioni and colleagues propose a realization of this concept. Specifically, they show that by limiting the coherent delocalization of energy to a restricted number of pigments within a cell, noise can produce a "hybrid quantum-classical" regime that optimizes energy transfer in terms of both speed and distance.

Beyond molecular biomimetic architectures, these ideas might find application in other classes of excitonic structures, such as materials based on semiconductor quantum dots that are being investigated for quantum information applications. These self-assembled quantum-dot-based materials also have a modular architecture, with unit cells made up of packed dimers of strongly interacting quantum dots. Previous work has shown that in these architectures, the coherent delocalization of excitation within the dimers may produce an energy structure similar to that of modular photosynthetic compounds [9]. These similarities bode well for the extension of the quantum design principles derived by Mattioni and colleagues to these quantum-dot-based materials.

Elisabetta Collini: Department of Chemical Science, University of Padua, Padua, Italy

\section{REFERENCES}

1. G. Ciamician, "The photochemistry of the future," Science 36, 385 (1912).

2. G. D. Scholes et al., "Lessons from nature about solar light harvesting," Nat. Chem. 3, 763 (2011).

3. A. H. Proppe et al., "Bioinspiration in light harvesting and catalysis," Nat. Rev. Mater. 5, 828 (2020).

4. A. Mattioni et al., "Design principles for long-range energy transfer at room temperature," Phys. Rev. X 11, 041003 (2021).

5. J. Cao et al., "Quantum biology revisited," Sci. Adv. 6, eaaz4888 (2020).

6. H. van Amerongen, R. van Grondelle, and L. Valkunas, Photosynthetic Excitons (World Scientific, Singapore, 2000).

7. M. Escalante et al., "Long-range energy propagation in nanometer arrays of light harvesting antenna complexes," Nano Lett. 10, 1450 (2010).

8. M. B. Plenio and S. F. Huelga, "Dephasing-assisted transport: quantum networks and biomolecules," New J. Phys. 10, 113019 (2008).

9. E. Collini et al., "Room-temperature inter-dot coherent dynamics in multilayer quantum dot materials," J. Phys. Chem. C 124, 16222 (2020). 